\title{
Clinical Significance of the HHLA2 Protein in Hepatocellular Carcinoma and the Tumor Microenvironment
}

\author{
Min Luo' \\ Yan Lin' \\ Rong Liang' \\ Yongqiang $\mathrm{Li}^{\prime}$ \\ Lianying $\mathrm{Ge}^{2}$ \\ 'Department of Oncology, Guangxi \\ Medical University Cancer Hospital, \\ Nanning, Guangxi, 53002I, People's \\ Republic of China; ${ }^{2}$ Department of \\ Endoscopy, Guangxi Medical University \\ Cancer Hospital, Nanning, Guangxi, \\ 53002I, People's Republic of China
}

Correspondence: Yongqiang $\mathrm{Li}$ Department of Oncology, Guangxi Medical University Cancer Hospital, Nanning, 53002I, People's Republic of China

Email lyq702702@I26.com

Lianying $\mathrm{Ge}$

Department of Endoscopy, Guangxi

Medical University Cancer Hospital,

Nanning, 53002I, People's Republic of

China

Email gxgly@hotmail.com
Background: The protein "human endogenous retrovirus H long terminal repeat-associating 2" (HHLA2), a member of the B7 family, has been linked to cancer progression and immune responses. However, its functional role in hepatocellular carcinoma (HCC) remains unknown.

Methods: Bioinformatics was used to examine the potential roles of HHLA2 in HCC and the molecular pathways involved. Expression of HHLA2 and PD-L1 as well as the density of tumor-infiltrating lymphocytes (TILs) in tumoral areas were evaluated by immunohistochemistry and hematoxylin-eosin staining of 202 resected human HCC samples. Potential correlations of HHLA2 expression with pathological characteristics or prognosis of HCC patients were explored. Different types of immune microenvironment in HCC were defined based on HHLA2 expression and TIL density.

Results: High HHLA2 levels in HCC correlated with more advanced clinical cancer stage $(P=0.040)$, multiple tumors $(P=0.044)$, poor tumor differentiation $(P=0.048)$, microvascular invasion $(P=0.011)$ and hepatic capsule invasion $(P=0.047)$. HHLA2 levels correlated significantly with density of TILs, but not with PD-L1 levels. High HHLA2 levels were associated with worse prognosis. Intermediate and high TIL densities were independent predictors of better prognosis. Tumor microenvironments with type I (HHLA2 - high TILs + ) or type IV (HHLA2 - low TILs + ) were associated with better prognosis.

Conclusion: HHLA2 level can independently predict worse prognosis and affect the tumor microenvironment in HCC, which may help guide immunotherapy against the cancer.

Keywords: hepatocellular carcinoma, HHLA2, tumor-infiltrating lymphocyte, tumor microenvironment

\section{Introduction}

Hepatocellular carcinoma (HCC) is one of the most common malignant tumors worldwide and is considered a major cause of cancer-related death. ${ }^{1}$ Most HCC cases are diagnosed at advanced stages, when prognosis is poor. Treatment options for advanced stages of HCC are often limited. The multi-target kinase inhibitor Sorafenib, as the standard first-line treatment, can enhance survival of patients with advanced HCC, but even so, median survival remains only 10.7 months and the drug is effective in only $2 \%$ of patients, yet it can trigger adverse events in as many as $80 \%$ of patients. ${ }^{2}$ Immunotherapy has opened up new possibilities against HCC: antibodies against Programmed cell death-1 (PD-1)/programmed death ligand-1 (PD-L1) act as immune checkpoint inhibitors and can achieve objective response rates of more than $20 \%$ and 30 month survival rates of $44 \%{ }^{3}$ 
While levels of PD-L1 on tumor cells can predict prognosis and response to anti-PD-1/PD-L1 immunotherapy, not all patients with PD-L1-positive tumors respond to the treatment. ${ }^{4-6}$ Another important factor may be the density of tumor-infiltrating lymphocytes (TILs), which, like PD-L1 levels in the tumor microenvironment, is associated with response to such therapy. ${ }^{5,7}$ TILs are important components of the tumor microenvironment, which influences the progression of malignant tumors and the host's anti-tumor immune response. ${ }^{8}$ In ovarian, breast, esophageal and colorectal cancers, TILs can inhibit tumor progression, affect the prognosis of patients, and predict the efficacy of tumor immunotherapy. ${ }^{9-12}$ A retrospective study of 163 patients with HCC linked high numbers of TILs, mainly T cells, with lower rate of 5-year recurrence and longer overall survival (OS). ${ }^{13}$

The protein "human endogenous retrovirus $\mathrm{H}$ long terminal repeat-associating 2" (HHLA2), a newly discovered member of the B7 family, co-stimulates and cosuppresses $\mathrm{T}$ cell proliferation and function, as well as the production of cytokines. ${ }^{14,15}$ HHLA2 can also promote angiogenesis and promote tumor growth and metastasis. HHLA2 is highly expressed in various malignant tumor tissues, and it has been linked to tumor progression and metastasis. ${ }^{16-22}$ In our preliminary study, we found that HHLA2 mRNA and protein levels were higher in HCC tissues than in para-carcinoma or normal liver tissues, and that higher HHLA2 expression was significantly associated with clinical stage, tumor differentiation and invasion of adjacent structures. In order to explore the potential usefulness of HHLA2 as a prognostic indicator or immunotherapy target in HCC, we explored potential associations of its expression with prognosis and characteristics of the tumor microenvironment.

\section{Methods}

\section{Bioinformatics Analysis}

The LinkedOmics website (http://www.linkedomics.org/ login.php) is an open web portal for multi-omics data [The Cancer Genome Atlas (TCGA) Research Network] that includes 32 cancers. ${ }^{23}$ We extracted RNA-sequencing data in the "TCGA-LIHC" dataset and applied Pearson correlation tests. Genes related to HHLA2 expression in HCC were identified using the LinkFinder module. Based on these co-expressed genes, Gene Ontology (GO) analysis and Kyoto Encyclopedia of Genes and Genomes
(KEGG) pathway enrichment analysis were performed using the LinkInterpreter module.

\section{Study Population and Tissue Samples}

There were 202 samples collected from patients who were initially treated in the Department of Hepatobiliary Surgery at Guangxi Medical University Cancer Hospital between January 2014 and December 2015. All underwent surgical resection, they had been diagnosed with HCC based on pathology, and their clinicopathological and follow-up data were complete. None of the patients received chemotherapy, targeted therapy or immunotherapy, nor did they have autoimmune diseases or tumors in other organs. All HCC tissues were taken from the primary tumor while avoiding areas of necrosis and inflammation, and were fixed with $10 \%$ neutral-buffered formalin, then embedded in paraffin. Detailed clinicopathologic characteristics of the patients are shown in Table 1. The last follow-up was December 31, 2019, and the median follow-up time was 37.2 months.

All patients provided written informed consent for participation in this study, which was approved by the Ethics Committee of Guangxi Medical University Cancer Hospital. All procedures involving human participants followed the ethical standards of the 1964 Helsinki Declaration and its later amendments.

\section{Histological Evaluation of TIL Density}

Each paraffin-embedded tissue was sectioned 3-4 $\mu \mathrm{m}$ thick, stained with hematoxylin-eosin (H\&E), then examined at magnifications of $\times 10$ and $\times 20$ by light microscopy. Average density of TILs was defined as the proportion of intratumoral stromal area occupied by mononuclear inflammatory cells. The intratumoral stromal area was defined to include both "central tumor" and "invasive margin", based on the guidelines of the International ImmunoOncology Biomarkers Working Group for solid tumors. ${ }^{24,25}$ The "central tumor" was defined as the central tumor tissue surrounded by the "invasive margin", which was defined as a zone $1 \mathrm{~mm}$ wide centered on the border of the malignant cells with the host tissue. Low TIL density was defined as less than $10 \%$; intermediate TIL density, $10-40 \%$; and high TIL density, as more than $40 \%$.

\section{Immunohistochemical Staining}

Paraffin-embedded tissue sections ( $4 \mu \mathrm{m}$ thick) were deparaffinized in xylene and hydrated through a graded ethanol series descending to distilled water. Subsequently, antigen retrieval was performed using microwave pre- 
Table I Correlations Between HHLA2 Protein Expression and Clinicopathological Characteristics in HCC Patients ( $\mathrm{n}=202$ )

\begin{tabular}{|c|c|c|c|c|c|}
\hline \multicolumn{2}{|l|}{ Clinical Character } & \multirow{3}{*}{$\begin{array}{c}\text { Number of Cases (\%) } \\
\begin{array}{c}174(86.1 \%) \\
28(13.9 \%)\end{array}\end{array}$} & \multicolumn{2}{|c|}{ HHLA2 } & \multirow{3}{*}{\begin{tabular}{|l}
$\mathbf{P}$ value \\
0.267
\end{tabular}} \\
\hline & & & Low Expression & High Expression & \\
\hline Sex & $\begin{array}{l}\text { Male } \\
\text { Female }\end{array}$ & & $\begin{array}{l}88(50.6 \%) \\
\text { II (39.3\%) }\end{array}$ & $\begin{array}{l}86(49.4 \%) \\
17(60.7 \%)\end{array}$ & \\
\hline Age(years) & $\begin{array}{l}>60 \\
\leq 60\end{array}$ & $\begin{array}{c}36(17.8 \%) \\
166(82.2 \%)\end{array}$ & $\begin{array}{l}17(47.2 \%) \\
82(49.4 \%)\end{array}$ & $\begin{array}{l}19(52.8 \%) \\
84(50.6 \%)\end{array}$ & 0.813 \\
\hline $\mathrm{HBsAg}$ & $\begin{array}{l}\text { Negative } \\
\text { Positive }\end{array}$ & $\begin{array}{c}24(11.9 \%) \\
178(88.1 \%)\end{array}$ & $\begin{array}{l}13(54.2 \%) \\
86(48.3 \%)\end{array}$ & $\begin{array}{l}\text { II (45.8\%) } \\
92(51.7 \%)\end{array}$ & 0.590 \\
\hline AFP level & $\begin{array}{l}>400 \mathrm{ng} / \mathrm{mL} \\
\leq 400 \mathrm{ng} / \mathrm{mL}\end{array}$ & $\begin{array}{l}100(49.5 \%) \\
102(50.5 \%)\end{array}$ & $\begin{array}{l}48(48 \%) \\
51(50 \%)\end{array}$ & $\begin{array}{l}52(52 \%) \\
51(50 \%)\end{array}$ & 0.776 \\
\hline Edmondson grade & $\begin{array}{c}\text { I-II } \\
\text { III-IV }\end{array}$ & $\begin{array}{c}87(43.1 \%) \\
115(56.9 \%)\end{array}$ & $\begin{array}{l}49(56.3 \%) \\
50(43.5 \%)\end{array}$ & $\begin{array}{l}38(43.7 \%) \\
65(56.5 \%)\end{array}$ & 0.048 \\
\hline Tumor size & $\begin{array}{l}>5 \mathrm{~cm} \\
\leq 5 \mathrm{~cm}\end{array}$ & $\begin{array}{l}|2|(59.9 \%) \\
8 \mid(40.1 \%)\end{array}$ & $\begin{array}{l}56(46.3 \%) \\
43(53.1 \%)\end{array}$ & $\begin{array}{l}65(53.7 \%) \\
38(46.9 \%)\end{array}$ & 0.343 \\
\hline Tumor number & $\begin{array}{c}\text { Single } \\
\text { Multiple }\end{array}$ & $\begin{array}{c}145(71.8 \%) \\
57(28.2 \%)\end{array}$ & $\begin{array}{l}77(53.1 \%) \\
22(38.6 \%)\end{array}$ & $\begin{array}{l}68(46.9 \%) \\
35(61.4 \%)\end{array}$ & 0.044 \\
\hline Satellite nodules & $\begin{array}{l}\text { No } \\
\text { Yes }\end{array}$ & $\begin{array}{c}167(82.7 \%) \\
35(17.3 \%)\end{array}$ & $\begin{array}{l}84(50.3 \%) \\
15(42.9 \%)\end{array}$ & $\begin{array}{l}83(49.7 \%) \\
20(57.1 \%)\end{array}$ & 0.423 \\
\hline Microvascular invasion & $\begin{array}{l}\text { No } \\
\text { Yes }\end{array}$ & $\begin{array}{c}80(39.6 \%) \\
122(60.4 \%)\end{array}$ & $\begin{array}{c}48(60 \%) \\
51(41.8 \%)\end{array}$ & $\begin{array}{c}32(40 \%) \\
71(58.2 \%)\end{array}$ & 0.011 \\
\hline Liver cirrhosis & $\begin{array}{l}\text { No } \\
\text { Yes }\end{array}$ & $\begin{array}{c}104(51.5 \%) \\
98(48.5 \%)\end{array}$ & $\begin{array}{l}54 \text { (51.9\%) } \\
45 \text { (45.9\%) }\end{array}$ & $\begin{array}{l}50(48.1 \%) \\
53(54.1 \%)\end{array}$ & 0.394 \\
\hline CNLC stage & $\begin{array}{l}\text { I } \\
\text { II } \\
\text { III }\end{array}$ & $\begin{array}{l}98(48.5 \%) \\
45(22.3 \%) \\
59(29.2 \%)\end{array}$ & $\begin{array}{c}49(50 \%) \\
28(62.2 \%) \\
22(37.3 \%)\end{array}$ & $\begin{array}{c}49(50 \%) \\
17(37.8 \%) \\
37(62.7 \%)\end{array}$ & 0.040 \\
\hline Capsular invasion & $\begin{array}{l}\text { Negative } \\
\text { Positive }\end{array}$ & $\begin{array}{c}77(38.1 \%) \\
125(61.9 \%)\end{array}$ & $\begin{array}{c}44(57.1 \%) \\
55(44 \%)\end{array}$ & $\begin{array}{c}33(42.9 \%) \\
70(56 \%)\end{array}$ & 0.047 \\
\hline
\end{tabular}

Abbreviations: $\mathrm{HCC}$, hepatocellular carcinoma; HHLA2, human endogenous retrovirus $\mathrm{H}$ long terminal repeat-associating 2; $\mathrm{HBs} A \mathrm{~g}$, hepatitis $B$ virus antigen; $\mathrm{AFP}$, alpha fetoprotein; CNLC, China liver cancer staging.

treatment in citrate-EDTA-buffer pH6.0 (ZSGB-BIO, Beijing, China) for $3 \mathrm{~min}$. The sections were then treated with $3 \%$ hydrogen peroxide for 10 minutes at room temperature to block endogenous peroxidase activity. Sections were incubated with rabbit polyclonal antibody against HHLA2 (dilution 1:200; \#ab214327, Abcam, Cambridge, UK) or rabbit monoclonal antibody against PD-L1 (dilution 1:400; \#ab205921, Abcam) for 1 hour at $37^{\circ} \mathrm{C}$. Then 
the PV-8000 Polymer Detection System for ImmunoHistological Staining (ZSGB-BIO, Beijing, China) was used according to the manufacturer's instructions, followed by Liquid DAB (Solarbio, Beijing, China) as a chromogen. Finally, sections were counterstained with hematoxylin and examined by light microscopy.

Stained sections were examined independently by two experienced investigators at $400 \times$ magnification, who assigned semi-quantitative immunoreactivity scores (IRS) based on the area of staining intensity and the percentage of positive cells. ${ }^{26}$ Staining intensity was scored as 0 (negative), 1 (weak), 2 (moderate), or 3 (strong). Percentage of positive cells was scored as 0 when none of the observed cells stained positive; 1 , when $<10 \%$ of observed cells were positive; $2,10-50 \%$ of cells; $3,51-80 \%$ of cells; or $4,>80 \%$ of cells. The IRS was evaluated in five visual fields from different areas of each tumor by multiplying the individual scores for staining intensity and percentage of positive cells. IRS could therefore vary from 0 to 12 points.

Cut-off values for stratifying tumors by IRS were determined using X-tile (New Haven, CT, USA). Tumors with IRS of at least 3 for PD-1 were considered positive for that protein. ${ }^{27}$ Tumors with IRS more than 3 for HHLA2 were considered to express "high HHLA2", while other tumors were considered to express "low HHLA2". ${ }^{28}$

\section{Statistical Analysis}

Data were analyzed using SPSS 25.0 (Chicago, IL, USA). Potential relationships between HHLA2 expression and clinicopathological characteristics were explored using a chi-squared test. Potential relationships of HHLA2 expression with TILs or PD-L1 expression were explored using Spearman analysis. Survival curves for patients stratified along different criteria were generated using the Kaplan-Meier method and compared using the Log rank test. Univariate and multivariate Cox proportional hazard regression was performed to identify factors associated with survival. $P<0.05$ was considered statistically significant.

\section{Results}

Bioinformatic Analysis of HHLA2 in HCC The gene expression profile of the 371 patients with HCC from the TCGA database was analyzed using LinkedOmics, identifying 19,922 differentially expressed genes (DEGs) related to HHLA2 expression. DEGs were depicted on volcano plots and heat maps (Figures 1A and $\mathrm{B})$, identifying the three genes most closely related to HHLA2 expression: TFF2, FABP6, and PPP1R14D. GO analysis showed that these three DEGs were significantly associated with immune-response biological processes, such as T cell activation, lymphocyte activation, and cell surface receptor signaling pathways that activate or regulate immune responses (Figure 1C). KEGG pathway analysis showed that the immune-related pathways of those DEGs were enriched mainly in Fc gamma R-mediated phagocytosis and signaling pathways involving TNF, Tolllike receptors, and NOD-like receptors (Figure 1D).

\section{Correlations Between HHLA2 Expression and Clinicopathology of HCC Patients}

Immunohistochemical staining detected HHLA2 on the membrane of tumor cells. Of the 202 patients examined, $103(51.0 \%)$ showed high HHLA2 expression, while 99 $(49.0 \%)$ showed low expression (Figures 2A and B). HHLA2 protein expression was higher with more advanced clinical cancer stage $(P=0.040)$, multiple tumors $(P=0.044)$, poorly differentiated tumors $(P=0.048)$, tumors with microvascular invasion $(P=0.011)$ or tumors with hepatic capsule invasion and $P=0.047$ ) (Table 1). These results suggest that HCC involving high HHLA2 expression is more aggressive.

No significant association was found between HHLA2 expression and sex, age, serum alpha-fetoprotein (AFP), tumor size, hepatitis B virus (HBV) infection, liver cirrhosis, or satellite nodules.

\section{PD-LI Expression and TILs in HCC Tissues}

Immunohistochemical staining detected PD-L1 on the membrane of tumor cells (Figures 2C and D). Of the 202 patients, $66(32.7 \%)$ were positive for PD-L1, while 136 (65.3\%) were negative for PD-L1. We observed that TILs, which appeared as mononuclear lymphocytes with round, hyperchromatic nuclei and blue-gray cytoplasm, were mostly enriched on the invasion edge of tumors (Figure 2E to G). Scattered TILs were observed in the tumor center. The density of TILs in HCC tissues was graded as low in 31 patients $(15.3 \%)$, intermediate in 107 cases $(53.0 \%)$, and high in $64(31.7 \%)$. 


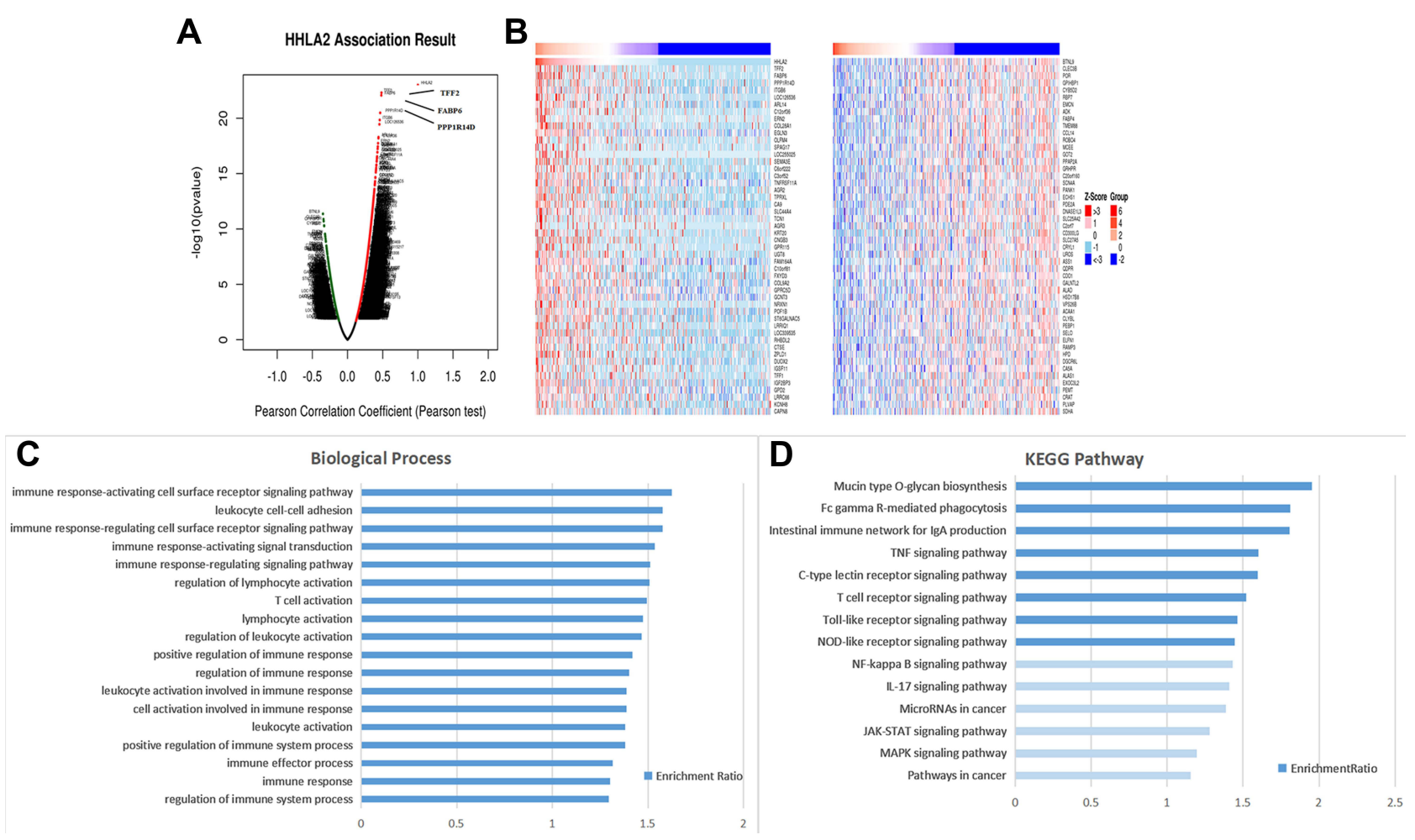

Figure I LinkedOmics analysis to identify differentially expressed genes related to HHLA2 in HCC. Volcano plot (A) of differentially expressed genes, showing three genes most closely related with HHLA2 expression: TFF2, FABP6, PPPIR 4DD. Heat map (B) of the derived gene-expression signature that correlated with HHLA2 expression. The bar plot of HHLA2 co-expression gene enrichment terms based on GO analysis (C) and KEGG pathway analysis (D). Dark blue indicates a false discovery rate $\leq 0.05$; light blue, a false discovery rate $>0.05$.

Abbreviations: HCC, hepatocellular carcinoma; HHLA2, human endogenous retrovirus H long terminal repeat-associating 2; GO, Gene Ontology; KEGG, Kyoto Encyclopedia of Genes and Genomes.

\section{Correlation of HHLA2 with TILs and PD-LI in HCC Tissues}

Spearman analysis showed that HHLA2 expression correlated negatively with TILs $\left(r_{\mathrm{s}}=-0.154, P=0.028\right.$; Table 2). However, HHLA2 expression did not correlate with PD-L1 expression $(P=0.057)$. As shown in Figures $3 \mathrm{~A}$ and $\mathrm{B}$, high HHLA2 expression was associated with worse OS in HCC patients with low TIL density $(P=0.009)$ and intermediate TIL density $(P=0.002)$. In contrast, HHLA2 expression did not seem to influence OS of HCC patients with high TIL density $(P=0.191$, Figure 3C). Our data suggest that as TIL density increases, high HHLA2 expression exerts less influence on prognosis of HCC patients.

\section{Prognostic Significance of HHLA2, TILs and PD-LI in HCC}

Survival analyses of patients stratified according to HHLA2 expression, TIL density, and PD-L1 expression are shown in Figure 4A-F. The 103 patients with high
HHLA2 showed median disease-free survival (DFS) and OS of 8.4 and 30.5 months, significantly worse than the 46.6 and 50.6 months for the 99 patients with low HHLA2 (both $P<0.001$ ). The 171 patients with high or intermediate TIL density showed respective median DFS of 50.6 and 10.8 months and OS of 52.4 and 40.8, significantly better the 3.3 and 8.5 months for the 31 patients with low TIL density (all $P<0.001$ ). Patients positive for PD-L1 had significantly shorter median OS (36.0 months) than patients negative for PD-L1 (49.8 months, $P=0.023$ ), but DFS did not differ significantly between the two groups $(P=0.259)$.

Our univariate analysis identified the following factors as significantly correlated with OS (Table 3): AFP, tumor size, Edmondson grade, tumor number, satellite nodules, microvascular invasion, liver cirrhosis, clinical stage, capsular invasion, TILs, PD-L1, and HHLA2 expression. Multivariate analysis identified the following factors as independent prognostic factors: high HHLA2 [hazard ratio (HR) 2.80, $P<0.001$ ], microvascular invasion (HR $1.87, \mathrm{P}=0.034)$, tumor size $>5 \mathrm{~cm}(\mathrm{HR} 3.06, P<0.001)$, 

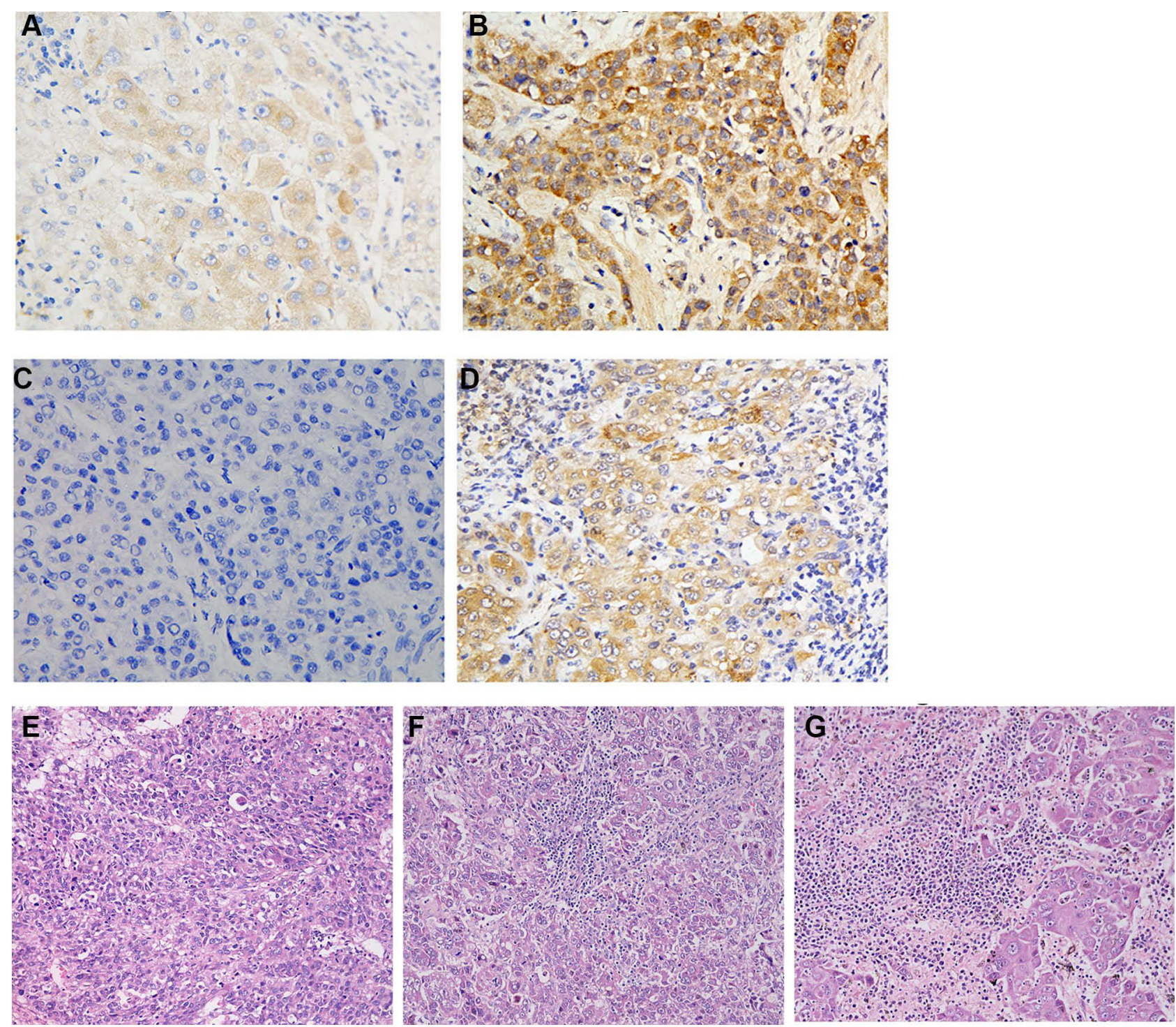

Figure 2 Expression of HHLA2 and PD-LI and density of TILs in HCC tissues. Representative micrographs of low HHLA2 expression (A) and high HHLA2 expression (B) within HCC tumors. Representative micrographs of negative PD-LI expression (C) and positive PD-LI expression (D) within HCC tumors. Representative H\&E staining of TILs in HCC tissues. Samples were graded as showing low TIL density (E), intermediate TIL density (F) or high TIL density (G).

Abbreviations: HCC, hepatocellular carcinoma; HHLA2, human endogenous retrovirus $\mathrm{H}$ long terminal repeat-associating 2; PD-LI, programmed death ligand-I; TILs, tumor-infiltrating lymphocytes.

multiple tumor number $(\mathrm{HR} 1.82, \mathrm{P}=0.007)$. Intermediate or high TIL density (HR $0.21, P<0.001$ ) were identified as independent predictors of better prognosis.

\section{Correlation Between Tumor}

Microenvironment and Prognosis in HCC Our finding of significant correlations among HHLA2 expression, TIL density and prognosis led us to examine associations between the tumor microenvironment and prognosis. Based on Teng et $\mathrm{al}^{29}$ we defined four types of tumor microenvironment: type I ("adaptive immune resistance", HHLA2 - high TILs +), which lacked HHLA2 and show intermediate or high TIL density, was observed in 77 of the $202 \mathrm{HCC}$ tissues (38.1\%); type II ("immunologic ignorance", HHLA2 - low TILs -), which lacked HHLA2 and showed low TIL density, was observed in 10 cases (5.0\%); type III ("intrinsic induction", HHLA2 - high TILs -), which lacked HHLA2 and showed intermediate or high TIL density, was observed in 26 cases (12.9\%); and type IV ("tolerance", HHLA2 - low TILs +), which lacked HHLA2 and low TIL density, was observed in 89 cases $(44.0 \%)$. 
Table 2 Correlation of HHLA2 Expression with TILs and PD-LI in HCC Tissues

\begin{tabular}{|c|c|c|c|c|c|c|}
\hline \multicolumn{2}{|c|}{ Variables } & \multirow[t]{3}{*}{ Number } & \multicolumn{2}{|c|}{ HHLA2 } & \multirow[t]{3}{*}{$r_{s}$} & \multirow[t]{3}{*}{$P$ value } \\
\hline & & & Low & High & & \\
\hline & & & $(n=99)$ & $(n=103)$ & & \\
\hline \multirow[t]{4}{*}{ TILs } & & & & & -0.154 & 0.028 \\
\hline & Low & 36 & 10 & 26 & & \\
\hline & Intermediate & 102 & 54 & 48 & & \\
\hline & High & 64 & 35 & 29 & & \\
\hline \multirow[t]{3}{*}{ PD-LI } & & & & & 0.134 & 0.057 \\
\hline & Negative & 136 & 73 & 63 & & \\
\hline & Positive & 66 & 26 & 40 & & \\
\hline
\end{tabular}

Abbreviations: $\mathrm{HCC}$, hepatocellular carcinoma; HHLA2, human endogenous retrovirus $\mathrm{H}$ long terminal repeat-associating 2; PD-LI, programmed death ligand-I; TILs, tumor infiltrating lymphocytes.

The four types of tumor microenvironment were associated with different OS and DFS (Figure 5). Prognosis was significantly better for patients with type I or IV tumor microenvironments, who had respective median DFS of 15.8 and 49.2 months and respective median OS of 49.5 and 51.3 months, than for patients with type II or III tumor microenvironments, who had respective median DFS of 8.8 and 2.3 months and median OS of 24.2 and 7.6 months (all $P<$ 0.001). Our results suggest that HCC patients with type I or IV tumor microenvironments are more likely to have better prognosis, probably because of stronger anti-tumor immune reactions.

\section{Discussion}

The tumor microenvironment is influential in tumorigenesis because it harbors tumor cells that interact with surrounding cells through the circulatory and lymphatic systems, and these interactions influence the development and progression of cancer. ${ }^{30}$ Anti-tumor immunotherapy, which aims to subvert tumor immunosuppression and enhance the body's anti-tumor immune response, has become the new focus of tumor therapy in recent years. In particular, antibodies against CTLA4 and PD-1/PD-L1 act as immune checkpoint inhibitors to restore $\mathrm{T}$ cell activity and induce robust anti-tumor immune responses. ${ }^{31}$

$\mathrm{HCC}$ is one of the most prevalent life-threatening malignancies, ranking as the sixth most frequently diagnosed cancer, and the fourth-leading cause of cancer-related death worldwide. $^{32}$ Studies have confirmed that $90 \%$ of HCC preferentially develops in a background of chronic liver damage and inflammation. Chronic liver damage alters the liver microenvironment and may help cancer cells evade immune surveillance by reprogramming the inflammatory environment, leading to inflammation-associated tumor formation. ${ }^{33}$ Anti-tumor immune responses require activation of $\mathrm{T}$ cells and subsequent recognition and killing of tumor cells by effector T cells. Molecules in the B7-CD28 family, as secondary signals of $\mathrm{T}$ cell activation, are crucial in $\mathrm{T}$ cell-mediated immune responses. The most widely used negative co-stimulatory molecules are B7.2 (CTLA-4), B7.1 (PD-1), and B7-H1 (PD-L1), which can be quite effective against advanced $\mathrm{HCC}$, providing a new direction for its treatment. Therefore, attention has been turned to the role of co-stimulatory or co-inhibitory molecules of the B7 family and their corresponding receptors in tumor immune regulation.

HHLA2, a newly described member of the B7 immunoglobulin superfamily, plays an important role in the adaptive immune system. HHLA2 was first discovered in the long terminal repeats of the HERV in the human genome, ${ }^{34}$ and its overexpression in the tumor microenvironment can inhibit T cell-mediated anti-tumor response, impair immune surveillance and promote tumor immune invasion. ${ }^{35}$ HHLA2 is widely expressed in a variety of malignant
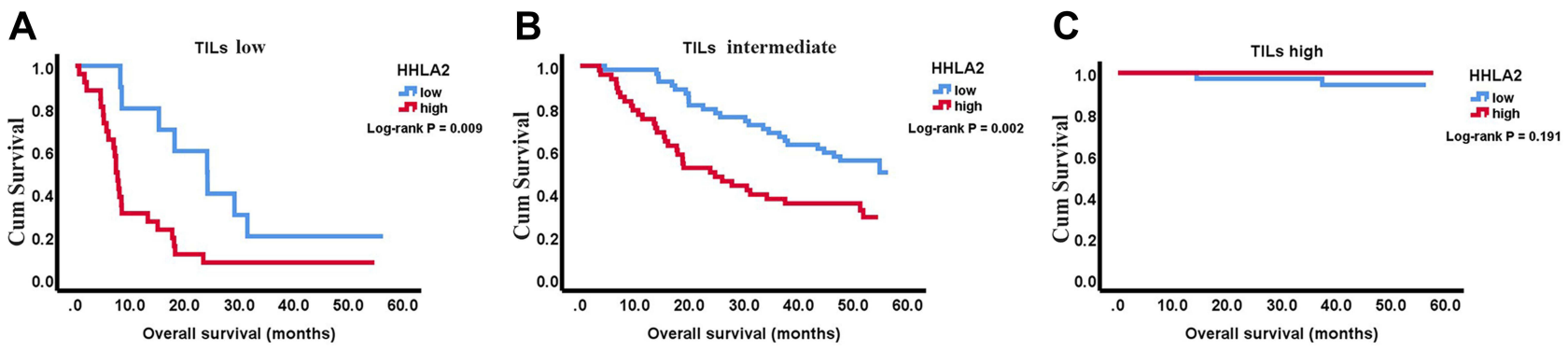

Figure 3 Kaplan-Meier survival curves showing OS of patients with HCC according to HHLA2 expression based on TILs. High HHLA2 expression was associated with worse OS in HCC patients with low TIL density (A) and intermediate TIL density (B). HHLA2 expression was not associated with OS of HCC patients with high TIL density (C).

Abbreviations: HCC, hepatocellular carcinoma; HHLA2, human endogenous retrovirus $\mathrm{H}$ long terminal repeat-associating 2; TILs, tumor-infiltrating lymphocytes; OS, overall survival. 
A

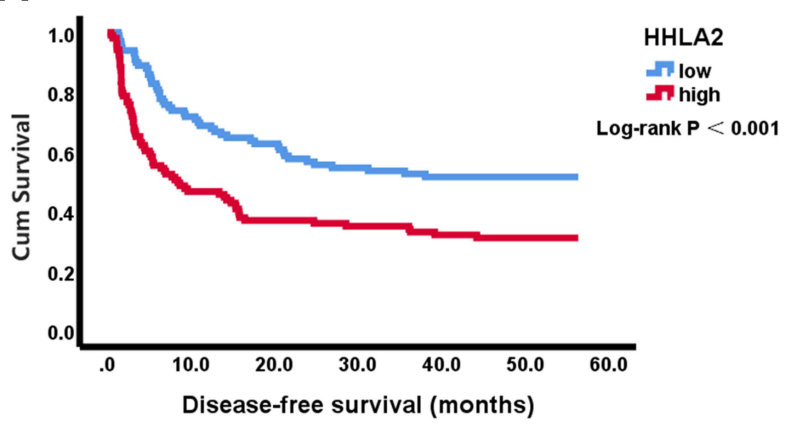

\section{C}

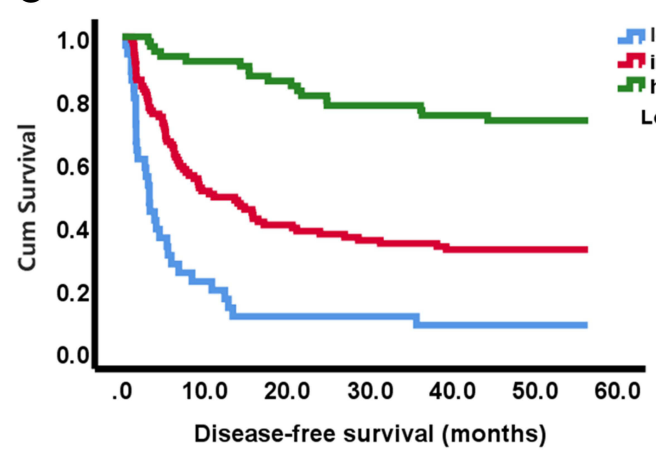

E

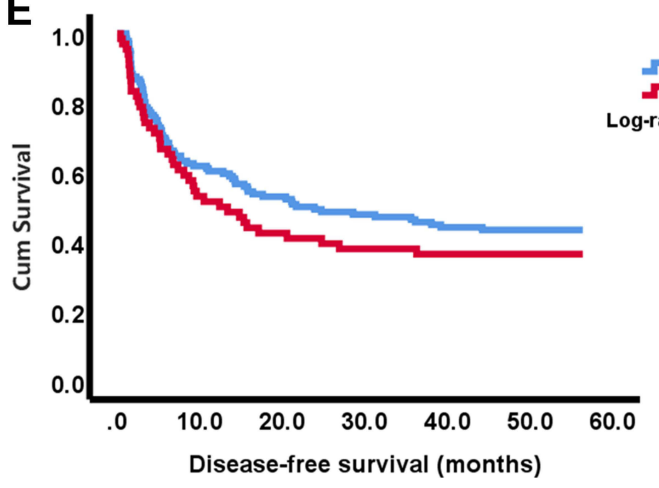

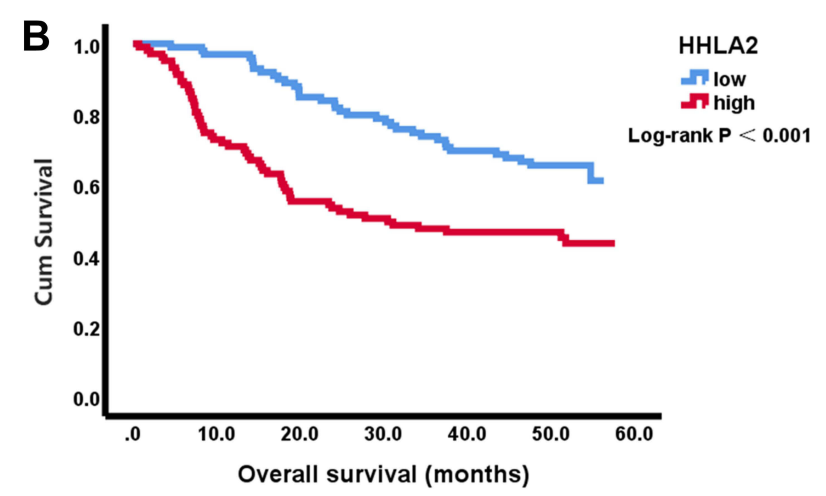

D

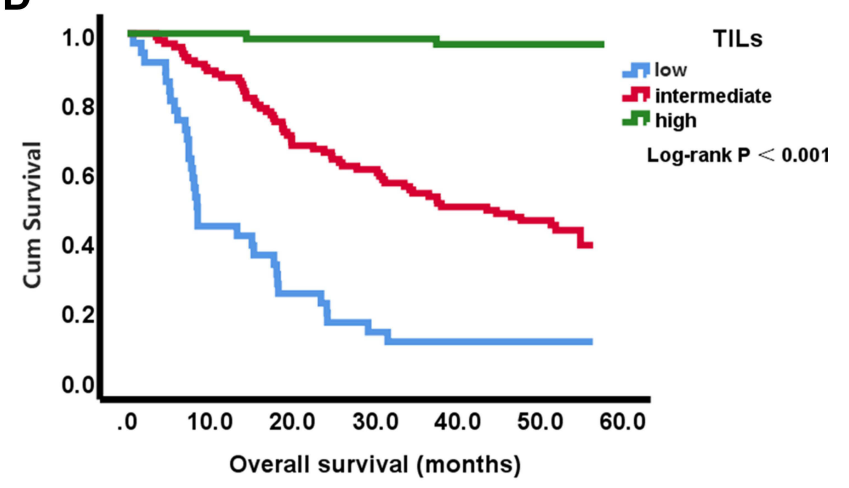

$\mathbf{F}$

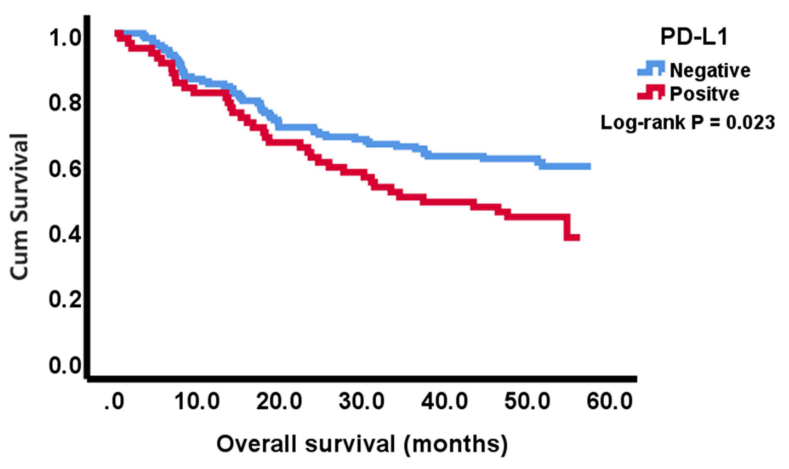

Figure 4 Kaplan-Meier survival curves showing DFS and OS of patients with HCC according to HHLA2 and PD-LI expression and density of TILs. High HHLA2 expression was significantly associated with poor DFS (A) and OS (B) in HCC. High and intermediate TIL density was significantly associated with better DFS (C) and OS (D) in HCC. PD-LI expression was not associated with DFS (E), but positive PD-LI expression was significantly associated with worse OS (F). Curves were compared using the Log rank test. Abbreviations: HCC, hepatocellular carcinoma; HHLA2, human endogenous retrovirus $\mathrm{H}$ long terminal repeat-associating 2; PD-LI, programmed death ligand-I; TILs, tumor-infiltrating lymphocytes; OS, overall survival; DFS, disease-free survival.

tumors, such as lung cancer, neuroendocrine tumors, and cervical adenocarcinoma. ${ }^{36-38}$ In our previously published work, we found that HHLA2 was highly expressed in HCC tissues, and was correlated with development and progression in HepG2 cells. ${ }^{39}$ We used the LinkOmics online database to analyze pathways in HCC that potentially involve HHLA2. We identified three genes closely related to HHLA2: TFF2, FABP6, and PPP1R14D. These genes have been linked to tumor occurrence and development in the digestive system. ${ }^{40-44} \mathrm{GO}$ analysis found that HHLA2 was involved mainly in the immune-related biological processes, and enriched KEGG pathways were Fc gamma R-mediated phagocytosis, TNF signaling pathways, and Toll-like receptor signaling pathways, all of which have been implicated in regulating the tumor microenvironment and promoting tumor development. ${ }^{45-48}$ In this way, both GO and KEGG pathway analysis suggest that HHLA2 is involved in immune regulatory pathways in HCC. These results may provide clues to explain our finding that HHLA2 influences the tumor microenvironment in HCC, given that the tumor microenvironment can reflect the host's anti-tumor immune response. 
Table 3 Cox model analysis for the association between the HHLA2 expression level and patients' clinical parameters

\begin{tabular}{|c|c|c|c|c|}
\hline \multirow[t]{2}{*}{ Variables } & \multicolumn{2}{|c|}{ Univariate Analysis } & \multicolumn{2}{|c|}{ Multivariate Analysis } \\
\hline & HR (95\% Cl) & $P$ value & HR (95\% CI) & $P$ value \\
\hline Gender (male: female) & $1.28(0.91 \sim 1.80)$ & 0.161 & & \\
\hline Age (> 60: $\leq 60$ years) & $1.35(0.77 \sim 2.39)$ & 0.299 & & \\
\hline HBsAg (positive: negative) & $0.68(0.34 \sim 1.36)$ & 0.277 & & \\
\hline AFP (>400ng/mL: $\leq 400 \mathrm{ng} / \mathrm{mL})$ & $0.64(0.52 \sim 0.80)$ & $<0.001$ & $1.47(0.87 \sim 2.48)$ & 0.151 \\
\hline Tumor size $(>5: \leq 5 \mathrm{~cm})$ & $0.21(0.12 \sim 0.36)$ & $<0.001$ & $3.06(I .24 \sim 4.1 I)$ & $<0.001$ \\
\hline Edmondson grade (I-II: III-IV) & $0.64(0.42 \sim 0.98)$ & 0.04 & $0.80(0.48 \sim 1.35)$ & 0.402 \\
\hline Tumor number (multiple: single) & $0.44(0.29 \sim 0.67)$ & $<0.001$ & $1.82(1.18 \sim 2.81)$ & 0.007 \\
\hline Satellite nodules (Yes: No) & $0.65(0.52 \sim 0.82)$ & $<0.001$ & $1.15(0.64 \sim 2.05)$ & 0.647 \\
\hline Microvascular invasion (Yes: No) & $0.48(0.37 \sim 0.63)$ & $<0.001$ & $1.87(1.05 \sim 3.35)$ & 0.034 \\
\hline Liver cirrhosis (Yes: No) & $1.18(0.78 \sim 1.78)$ & 0.424 & & \\
\hline CNLC stage (I: II+III) & $3.97(2.46 \sim 6.4 I)$ & $<0.001$ & $1.08(0.61 \sim 1.92)$ & 0.782 \\
\hline Capsular invasion (positive: negative) & $0.60(0.38 \sim 0.94)$ & 0.025 & $0.98(0.60 \sim 1.60)$ & 0.937 \\
\hline TILs (low: intermediate + high) & $0.22(0.14 \sim 0.35)$ & $<0.001$ & $0.21(0.15 \sim 0.31)$ & $<0.001$ \\
\hline PD-LI(positive: negative) & $0.79(0.64 \sim 0.97)$ & 0.027 & $1.77(1.10 \sim 2.88)$ & 0.084 \\
\hline HHLA2 expression (high: low) & $1.47(1.19 \sim 1.81)$ & $<0.001$ & $2.80(1.77 \sim 4.44)$ & $<0.001$ \\
\hline
\end{tabular}

Abbreviations: $\mathrm{HCC}$, hepatocellular carcinoma; $\mathrm{HHLA} 2$, human endogenous retrovirus $\mathrm{H}$ long terminal repeat-associating 2; $\mathrm{HBsAg}$, hepatitis $\mathrm{B}$ virus antigen; AFP, alpha fetoprotein; CNLC, China liver cancer staging; PD-LI, programmed death ligand-I; TILs, tumor infiltrating lymphocytes.

We further explored the expression and clinical significance of HHLA2 in HCC. HHLA2 was expressed mainly in the cell membrane and cytoplasm of HCC tissues, with little nuclear expression, and HHLA2 showed little heterogeneity within the tumor. Of the $202 \mathrm{HCC}$ tissues that we examined, $51 \%$ showed high HHLA2 expression, more than the $32.7 \%$ that showed PD-L1 expression. The high rate of high HHLA2 expression is consistent with the results of other studies, ${ }^{17,18,49,50}$ indicating that HHLA2 may be a target for immunotherapy. We found that high HHLA2 expression positively correlated with more advanced clinical cancer stage, multiple tumors, poorly differentiated tumors, microvascular invasion and hepatic capsule invasion. These results suggest that HCC involving high HHLA2 expression is more aggressive and metastatic, which in turn affects prognosis.
Correlations between TIL density and HHLA2 expression vary among different cancers. HHLA2 expression independently correlates with high TIL density in nonsmall cell lung cancer and renal cell carcinoma, ${ }^{17,49}$ but not in osteosarcoma. ${ }^{18}$ In lung cancer, HHLA2 expression significantly correlates with EGFR mutations, TIL density, and PD-L1 status, suggesting that HHLA2 may be an effective immunotherapy target in PD-L1-negative patients. ${ }^{17,51}$ In one study, an HHLA2-IG fusion protein did not interact with PD-L1, even though HHLA2 and PDL1 are both members of the B7 family and both can inhibit the activity of T cells. ${ }^{15}$ Another study showed no correlation between HHLA2 and PD-L1 levels. In contrast, a study of renal cell carcinoma found that patients expressing both HHLA2 and PD-L1 had the highest risk of tumor
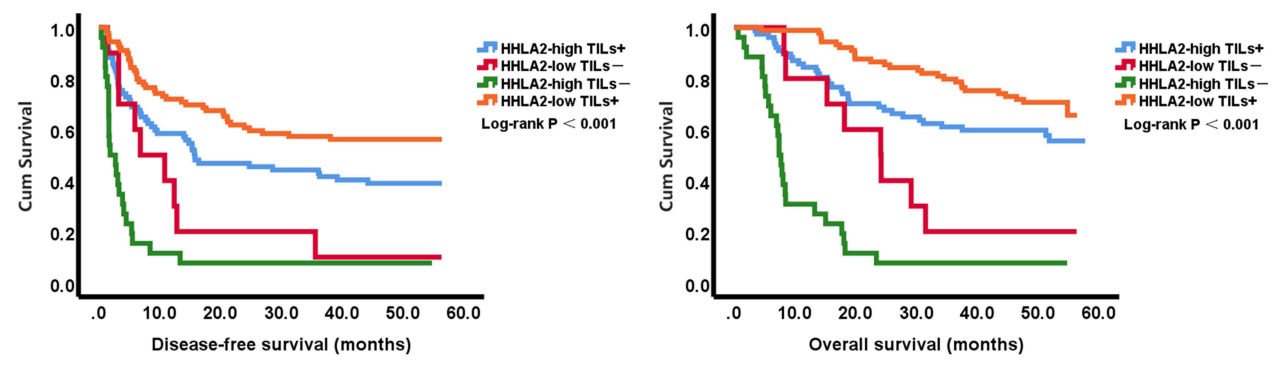

Figure 5 Kaplan-Meier survival curves showing DFS and OS of patients with HCC according tumor microenvironment based on HHLA2 expression and density of TILs. Patients with type I HCC (HHLA2 - high TILs +) or type IV HCC (HHLA2 - low TILs +) showed significantly better DFS and OS.

Abbreviations: HCC, hepatocellular carcinoma; HHLA2, human endogenous retrovirus $\mathrm{H}$ long terminal repeat-associating 2; TILs, tumor-infiltrating lymphocytes; OS, overall survival; DFS, disease-free survival. 
recurrence and death, and the risk correlated significantly with high TIL density. ${ }^{49}$ In our study of HCC, HHLA2 expression correlated significantly with TIL density, and patients with a high TIL density had better prognosis, consistent with the results of another study. ${ }^{52}$ We found that higher TIL density weakened the influence of high HHLA2 expression on prognosis. This may be because when there are few immune cells in the tumor microenvironment, HHLA2 is involved in immunosuppression and promotes tumor progression, supporting the idea that HHLA2 may be an immunotherapy target.

Studies have shown that high HHLA2 expression in malignant tumors may be an important prognostic indicator. Our study also showed that HCC patients with high HHLA2 expression were at higher risk of postoperative recurrence and poor clinical outcome. Our results and past studies suggest that HHLA2 is an important indicator of HCC recurrence, metastasis and poor prognosis. Indeed, univariate and multivariate analyses confirmed that high HHLA2 expression in HCC can significantly influence prognosis.

In a variety of malignant tumors, the combination of TIL density and PD-L1 expression in the tumor microenvironment can predict the efficacy of immunotherapy and guide the treatment of PD-1/PD-L1 inhibitors. The tumor microenvironment in most of our patients belonged to type I (38.1\%) or type IV (44.0\%), which were associated with longer OS. These results are consistent with the idea that high TIL density is associated with better prognosis. Conversely, we found that poor prognosis was associated with tumor microenvironments of type II and especially type III, indicating that high HHLA2 expression can inhibit TILs and generate immunosuppression. Additionally, it has recently been reported that HHLA2 engagement recruited its new inhibitory receptor KIR3DL3 to the immunological synapse and coinhibited CD8 $+\mathrm{T}$ and NK cell function and killing, protecting HHLA2+ tumor cells from immune attack. ${ }^{53,54}$ In summary, these results suggest that immunotherapy may be more suitable for HCC patients. These results also support the idea that HHLA2 expression can affect the tumor microenvironment in $\mathrm{HCC}$ and thereby influence prognosis.

There are few reports about the role and underlying molecular mechanisms of HHLA2 in HCC. In this study, we observed that high HHLA2 expression was associated with unfavorable prognosis in HCC and correlated with the TILs infiltration rather than PD-L1 expression. We also identified potential related-genes and pathways with HHLA2 in HCC through bioinformatics methods. These findings might indicate that HHLA2 is a target for HCC immunotherapy. However, there are some limitations in this study. First of all, selection bias is inevitable, because this was a retrospective study. A large prospective study is needed to verify these results. Second, we have only preliminary explored the correlation between HHLA2 and TILs, lack of immune cell subsets analysis for TILs. Third, specific molecular mechanisms involved in HHLA2 have not been investigated in our study and further research is required to be performed.

\section{Conclusion}

Our findings indicate that high HHLA2 expression can independently predict worse prognosis in hepatocellular carcinoma. HHLA2 may influence immune responses within the tumor microenvironment and thereby affect prognosis. These results may help guide immunotherapy of hepatocellular carcinoma.

\section{Acknowledgments}

This research was supported by the Youth Program of the Scientific Research Foundation of Guangxi Medical University Cancer Hospital (No. 2021-06). We are grateful to all patients who participated in this research.

\section{Disclosure}

The authors declare that they have no conflicts of interest related to this work.

\section{References}

1. Singal AG, Lampertico P, Nahon P. Epidemiology and surveillance for hepatocellular carcinoma: new trends. J Hepatol. 2020;72(2):250-261. doi:10.1016/j.jhep.2019.08.025

2. Llovet JM, Ricci S, Mazzaferro V, et al. Sorafenib in advanced hepatocellular carcinoma. $N$ Engl J Med. 2008;359(4):378-390. doi:10.1056/NEJMoa0708857

3. Yau T, Kang YK, Kim TY, et al. Nivolumab (NIVO) + ipilimumab (IPI) combination therapy in patients (pts) with advanced hepatocellular carcinoma (aHCC): results from CheckMate 040. J Clin Oncol. 2019;37:4012. doi:10.1200/JCO.2019.37.15_suppl.4012

4. Garon EB, Rizvi NA, Hui R, et al. Pembrolizumab for the treatment of non-small-cell lung cancer. $N$ Engl J Med. 2015;372(21):2018-2028. doi:10.1056/NEJMoa1501824

5. Herbst RS, Soria JC, Kowanetz M, et al. Predictive correlates of response to the anti-PD-L1 antibody MPDL3280A in cancer patients. Nature. 2014;515(7528):563-567. doi:10.1038/nature14011

6. Ansell SM, Lesokhin AM, Borrello I, et al. PD-1 blockade with nivolumab in relapsed or refractory Hodgkin's lymphoma. $N$ Engl J Med. 2015;372(4):311-319. doi:10.1056/NEJMoa1411087

7. Taube JM, Klein A, Brahmer JR, et al. Association of PD-1, PD-1 ligands, and other features of the tumor immune microenvironment with response to anti-PD-1 therapy. Clin Cancer Res. 2014;20 (19):5064-5074. doi:10.1158/1078-0432.CCR-13-3271 
8. Pagès F, Galon J, Dieu-Nosjean MC, et al. Immune infiltration in human tumors: a prognostic factor that should not be ignored Oncogene. 2010;29(8):1093-1102. doi:10.1038/onc.2009.416

9. Mahmoud SM, Paish EC, Powe DG, et al. Tumor-infiltrating CD8+ lymphocytes predict clinical outcome in breast cancer. J Clin Oncol. 2011;29(15):1949-1955. doi:10.1200/JCO.2010.30.5037

10. Laghi L, Bianchi P, Miranda E, et al. CD3+ cells at the invasive margin of deeply invading (pT3-T4) colorectal cancer and risk of post-surgical metastasis: a longitudinal study. Lancet Oncol. 2009;10 (9):877-884. doi:10.1016/S1470-2045(09)70186-X

11. Curiel TJ, Coukos G, Zou L, et al. Specific recruitment of regulatory $\mathrm{T}$ cells in ovarian carcinoma fosters immune privilege and predicts reduced survival. Nat Med. 2004;10(9):942-949. doi:10.1038/ nm1093

12. Taisuke Y, Yoshifumi B, Takatsugu I, et al. PD-L1 expression, tumor-infiltrating lymphocytes, and clinical outcome in patients with surgically resectes esophageal cancer. Ann Surg. 2019;269 (3):471-478. doi:10.1097/SLA.0000000000002616

13. Wada Y, Nakashima O, Kutami R, et al. Clinicopathological study on hepatocellular carcinoma with lymphocytic infiltration. Hepatology. 1998;27(2):407-414. doi:10.1002/hep.510270214

14. Zhu Y, Yao S, Iliopoulou BP, et al. B7-H5 costimulates human T cells via CD28H. Nat Commun. 2013;4:2043. doi:10.1038/ncomms3043

15. Zhao R, Chinai JM, Buhl S, et al. HHLA2 is a member of the B7 family and inhibits human CD4 and CD8 T-cell function. Proc Natl Acad Sci USA. 2013;110(24):9879-9884. doi:10.1073/pnas.1303524110

16. Janakiram M, Chinai JM, Fineberg S, et al. Expression, clinica significance, and receptor identification of the newest B7 family member HHLA2 protein. Clin Cancer Res. 2015;21(10):2359-2366. doi:10.1158/1078-0432.CCR-14-1495

17. Cheng H, Janakiram M, Borczuk A, et al. HHLA2, a new immune checkpoint member of the B7 family, is widely expressed in human lung cancer and associated with EGFR mutational status. Clin Cancer Res. 2017;23(3):825-832. doi:10.1158/1078-0432.CCR-15-3071

18. Koirala P, Roth ME, Gill J, et al. HHLA2, a member of the B7 family, is expressed in human osteosarcoma and is associated with metastases and worse survival. Sci Rep. 2016;6:31154. doi:10.1038/ srep3 1154

19. Lin G, Ye H, Wang J, et al. Immune checkpoint human endogenous retrovirus-H long terminal repeat-associating protein 2 is upregulated and independently predicts unfavorable prognosis in bladder urothelial carcinoma. Nephron. 2019;141(4):256-264. doi:10.1159/ 000495887

20. Chen L, Zhu D, Feng J, et al. Overexpression of HHLA2 in human clear cell renal cell carcinoma is significantly associated with poor survival of the patients. Cancer Cell Int. 2019;19:101. doi:10.1186/ s12935-019-0813-2

21. Chen $\mathrm{D}$, Chen $\mathrm{W}, \mathrm{Xu} \mathrm{Y}$, et al. Upregulated immune checkpoint HHLA2 in clear cell renal cell carcinoma: a novel prognostic biomarker and potential therapeutic target. J Med Genet. 2019;56 (1):43-49. doi:10.1136/jmedgenet-2018-105454

22. Zhu Z, Dong W. Overexpression of HHLA2, a member of the B7 family, is associated with worse survival in human colorectal carcinoma. Onco Targets Ther. 2018;11:1563-1570. doi:10.2147/ OTT.S160493

23. Vasaikar SV, Straub P, Wang J, Zhang B. LinkedOmics: analyzing multi-omics data within and across 32 cancer types. Nucleic Acids Res. 2018;46(D1):D956-D963. doi:10.1093/nar/gkx1090

24. Hendry S, Salgado R, Gevaert T, et al. Assessing tumor-infiltrating lymphocytes in solid tumors: a practical review for pathologists and proposal for a standardized method from the international immunooncology biomarkers working group: part 1: assessing the host immune response, TILs in invasive. Adv Anat Pathol. 2017;24(5):235-251. doi:10.1097/PAP.0000000000000162
25. Hendry S, Salgado R, Gevaert T, et al. Assessing tumor-infiltrating lymphocytes in solid tumors: a practical review for pathologists and proposal for a standardized method from the international immuno-oncology biomarkers working group: part 2: tILs in melanoma, gastrointestinal tract carcinomas, non-small cell lung carcinoma and mesothelioma, endometrial and ovarian carcinomas, squamous cell carcinoma of the head and neck, genitourinary carcinomas, and primary brain tumors. Adv Anat Pathol. 2017;24 (6):311-335. doi:10.1097/PAP.0000000000000161

26. McCarty KS Jr, Miller LS, Cox EB, et al. Estrogen receptor analyses. Correlation of biochemical and immunohistochemical methods using monoclonal antireceptor antibodies. Arch Pathol Lab Med. 1985;109 (8):716-721.

27. Jung HI, Jeong D, Ji S, et al. Overexpression of PD-L1 and PD-L2 is associated with poor prognosis in patients with hepatocellular carcinoma. Cancer Res Treat. 2017;49(1):246-254. doi:10.4143/ crt.2016.066

28. Camp RL, Dolled-Filhart M, Rimm DL. X-Tile: a new bio-informatics tool for biomarker assessment and outcome-based cut-point optimization. Clin Cancer Res. 2004;10(21):7252-7259. doi:10.1158/1078-0432.CCR-04-0713

29. Teng MW, Ngiow SF, Ribas A, Smyth MJ. Classifying cancers based on T-cell infiltration and PD-L1. Cancer Res. 2015;75 (11):2139-2145. doi:10.1158/0008-5472.CAN-15-0255

30. Arneth B. Tumor microenvironment. Medicina. 2019;56(1):15. doi:10.3390/medicina56010015

31. Mcdermott DF, Atkins MB. PD-1 as a potential target in cancer therapy. Cancer Med. 2013;2(5):662-673. doi:10.1002/cam4.106

32. Bray F, Ferlay J, Soerjomataram I, et al. Global Cancer Statistics 2018: GLOBOCAN estimates of incidence and mortality worldwide for 36 cancers in 185 countries. CA Cancer J Clin. 2018;68 (6):394-424. doi: $10.3322 /$ caac. 21492

33. Yang YM, Kim SY, Seki E. Inflammation and liver cancer: molecular mechanisms and therapeutic targets. Semin Liver Dis. 2019;39 (1):26-42. doi:10.1055/s-0038-1676806

34. Mager DL, Hunter DG, Schertzer M, Freeman JD. Endogenous retroviruses provide the primary polyadenylation signal for two new human genes (HHLA2 and HHLA3). Genomics. 1999;59 (3):255-263. doi:10.1006/geno.1999.5877

35. Janakiram M, Chinai JM, Zhao A, et al. HHLA2 and TMIGD2: new immunotherapeutic targets of the B7 and CD28 families. OncoImmunology. 2015;4(8):e1026534. doi:10.1080/ 2162402X.2015.1026534

36. Farrag MS, Ibrahim EM, El-Hadidy TA, et al. Human endogenous retrovirus-H long terminal repeat-associating protein 2 (HHLA2) is a novel immune checkpoint protein in lung cancer which predicts survival. Asian Pac J Cancer Prev. 2021;22(6):1883-1889. doi:10.31557/APJCP.2021.22.6.1883

37. Yuan Z, Gardiner JC, Maggi EC, et al. B7 immune-checkpoints as targets for the treatment of neuroendocrine tumors. Endocr Relat Cancer. 2021;28(2):135-149. doi:10.1530/ERC-20-0337

38. Byun JM, Cho HJ, Park HY, et al. The clinical significance of HERV-H LTR -associating 2 expression in cervical adenocarcinoma. Medicine. 2021;100(1):e23691. doi:10.1097/ MD.0000000000023691

39. Luo M, Xiong Y, Lin Y, et al. H Long Terminal Repeat-Associating 2 (HHLA2) is a biomarker of advanced stage hepatocellular carcinoma and promotes tumor cell development in vitro. Med Sci Monit. 2021;27:e930215. doi:10.12659/MSM.930215

40. Muenphon K, Limpaiboon T, Jearanaikoon P, et al. Amplification of chromosome 21q22.3 harboring trefoil factor family genes in liver fluke related cholangiocarcinoma is associated with poor prognosis. World J Gastroenterol. 2006;12(26):4143-4148. doi:10.3748/wjg. v12.i26.4143 
41. Lu Y, Ren J. Advance in trefoil factor family. World Chin J Digestol. 2003;11(18):2019-2021.

42. Zhang Y, Zhao X, Deng L, et al. High expression of FABP4 and FABP6 in patients with colorectal cancer. World J Surg Oncol. 2019;17(1):171. doi:10.1186/s12957-019-1714-5

43. Ohmachi T, Inoue H, Mimori K, et al. Fatty acid binding protein 6 is overexpressed in colorectal cancer. Clin Cancer Res. 2006;12 (17):5090-5095. doi:10.1158/1078-0432.CCR-05-2045

44. Eskandari E, Mahjoubi F, Motalebzadeh J. An integrated study on TFs and miRNAs in colorectal cancer metastasis and evaluation of three co-regulated candidate genes as prognostic markers. Gene. 2018;679:150-159. doi:10.1016/j.gene.2018.09.003

45. Dahan R, Sega E, Engelhardt J, et al. FcyRs modulate the anti-tumor activity of antibodies targeting the PD-1/PD-L1 axis. Cancer Cell. 2015;28(3):285-295. doi:10.1016/j.ccell.2015.08.004

46. Golay J, Da Roit F, Bologna L, et al. Glycoengineered CD20 antibody obinutuzumab activates neutrophils and mediates phagocytosis through CD16B more efficiently than rituximab. Blood. 2013;122 (20):3482-3491. doi:10.1182/blood-2013-05-504043

47. Wu Y, Zhou BP. TNF-alpha/NF-kappaB/Snail pathway in cancer cell migration and invasion. $B r \quad J$ Cancer. 2010;102(4):639-644. doi:10.1038/sj.bjc.6605530

48. Jang MK, Kim HS, Chung YH. Clinical aspects of tumor necrosis factor- $\alpha$ signaling in hepatocellular carcinoma. Curr Pharm Des. 2014;20(17):2799-2808. doi:10.2174/13816128113199990587
49. Zhou QH, Li KW, Chen X, et al. HHLA2 and PD-L1 co-expression predicts poor prognosis in patients with clear cell renal cell carcinoma. J Immunother Cancer. 2020;8(1):e000157. doi:10.1136/ jitc-2019-000157

50. Jing CY, Fu YP, Yi Y, et al. HHLA2 in intrahepatic cholangiocarcinoma: an immune checkpoint with prognostic significance and wider expression compared with PD-L1. J Immunother Cancer. 2019;7 (1):77. doi:10.1186/s40425-019-0554-8

51. Cheng HY, Borczuk A, Janakiram M, et al. Wide expression and significance of alternative immune checkpoint molecules, B7x and HHLA2, in PD-L1-negative human lung cancers. Clin Cancer Res. 2018;24(8):1954-1964. doi:10.1158/1078-0432.CCR-17-2924

52. Yoshito W, Osamuv N, Rumikob K, et al. Clinicopathological study on hepatocellular carcinoma with lymphocytic infiltration. Hepatology. 1998;27(2):407-414. doi:10.1002/hep.510270214

53. Campbell KS. Mystery checkpoint revealed: KIR3DL3 finally found a ligand in HHLA2. Cancer Immunol Res. 2021;9(2):128. doi:10.1158/2326-6066.CIR-20-0996

54. Wei Y, Ren X, Galbo PM, et al. KIR3DL3-HHLA2 is a human immunosuppressive pathway and a therapeutic target. Sci Immunol. 2021;6(61):eabf9792. doi:10.1126/sciimmunol.abf9792
Journal of Inflammation Research

\section{Publish your work in this journal}

The Journal of Inflammation Research is an international, peerreviewed open-access journal that welcomes laboratory and clinical findings on the molecular basis, cell biology and pharmacology of inflammation including original research, reviews, symposium reports, hypothesis formation and commentaries on: acute/chronic inflammation; mediators of inflammation; cellular processes; molecular

\section{Dovepress}

mechanisms; pharmacology and novel anti-inflammatory drugs; clinical conditions involving inflammation. The manuscript management system is completely online and includes a very quick and fair peerreview system. Visit http://www.dovepress.com/testimonials.php to read real quotes from published authors. 